\title{
CONTRACTION SEMIGROUPS FOR DIFFUSION WITH DRIFT
}

BY

R. SEELEY

\begin{abstract}
Recently Dodziuk, Karp and Li, and Strichartz have given results on existence and uniqueness of contraction semigroups generated by the Laplacian $\Delta$ on a manifold $M$; earlier, Yau gave related results for $L=\Delta+V$ for a vector field $V$. The present paper considers $L=\Delta-V-c$, with $c$ a real function, and gives conditions for (a) uniqueness of semigroups on the bounded continuous functions, (b) preservation of $C_{0}$ (functions vanishing at $\infty$ ) by the minimal semigroup, and (c) existence and uniqueness of contraction semigroups on $L^{p}(\mu), 1 \leqslant p<\infty$, for an arbitrary smooth density $\mu$ on $M$. The conditions concern $L \rho / \rho$, where $\rho$ is a smooth function, $\rho \rightarrow \infty$ as $x \rightarrow \infty$. They variously extend, strengthen, and complement the previous results mentioned above.
\end{abstract}

\section{Introduction. Consider an operator}

$$
L u=\Delta u-V u-c u,
$$

where $\Delta$ is the Laplacian on a noncompact Riemannian manifold $M, V$ is a vector field, and $c$ a function. (All coefficients are assumed reasonably smooth.) $L$ represents a diffusion with "drift" $V$ and "local dissipation" $c$. The evolution in time of an initial distribution $f$ is a solution of the Cauchy problem for the heat equation:

$$
\left\{\begin{array}{l}
\partial u / \partial t=L u, \quad t>0 \\
u=f \text { when } t=0 \\
u \text { continuous for } t \geqslant 0
\end{array}\right.
$$

The sign of $V$ in (1) has a simple interpretation: if $V(x) \cdot d u(x)>0$ then the stuff brought toward $x$ by $V$ is at a lower temperature than the stuff being taken away, thus causing a drop in the temperature $u$ at $x$.

If $c \geqslant 0$, equation (2) always has a "minimal solution" which can be obtained as follows (see Dodziuk [D] for more details). Represent $M$ as the union of an increasing sequence of compact manifolds with boundary $M_{n}$. Let $u_{n}$ be the solution of (2) on $M_{n}$ with $u_{n}=0$ on $\partial M_{n}$. This solution can be represented as

$$
u_{n}(t, x)=\int_{M_{n}} p_{n}(t, x, y) f(y) d v_{y},
$$

where $p_{n}$ is positive, $\int p_{n} d v_{y} \leqslant 1, \lim _{t \rightarrow 0^{+}} \int p_{n} d v_{y}=1$, and

$$
\partial p_{n} / \partial t=L_{x} p_{n}=L_{y}^{*} p_{n} \text {. }
$$

Received by the editors April 26, 1983 and, in revised form, August 2, 1983.

1980 Mathematics Subject Classification. Primary 35K05; Secondary 58G32. 
( $L_{x}$ indicates $L$ acting in the $x$ variable, and $L^{*}$ is the formal adjoint of $L$.) By the maximum principle and the monotone convergence theorem, the $p_{n}$ converge up to a function $p$ with $\int p d v_{y} \leqslant 1$, and (in the sense of distributions)

$$
\partial p / \partial t=L_{x} p=L_{y}^{*} p, \quad t>0 .
$$

Since $p$ satisfies the parabolic equation $2 p_{t}=L_{x} p+L_{x}^{*} p$, it is smooth in all variables for $t>0$. If $f$ is a bounded continuous function on $M(f \in \mathrm{BC}(M))$, then the function

$$
u(x, t)=\int p(t, x, y) f(y) d v_{y}
$$

solves the Cauchy problem (2). The map $f \rightarrow u(\cdot, t)$ defines a contraction semigroup on the space $\mathrm{BC}(M)$, with infinitesimal generator $L$ defined on an appropriate domain containing $C_{c}^{2}(M)$, the $C^{2}$ functions with compact support.

$\S 1$ considers uniqueness of the solution (3), and $\$ 2$ concerns the preservation of $C_{0}$ (continuous functions vanishing at $\infty$ ). $\$ 3$ considers the existence of contraction semigroups on $L^{p}, 1 \leqslant p<\infty$, generalizing some results of Strichartz [S]. The interesting condition there relates the divergence of $V$ to the local dissipation $c$. Specifically, Theorem 3 says: The operator $L$ is dissipative on $L^{p}$ if

$$
(1 / p) \operatorname{div} V \leqslant c .
$$

As $p \rightarrow \infty$ this reduces to the familiar condition $c \geqslant 0$. The condition is also necessary when $p=1$, or when $L=-V-c(\Delta=0)$. In any case, if it is uniformly violated on a sufficiently large set, then $L$ is not dissipative.

There are several results similar to those in $\S \S 1$ and 2. A standard reference (particularly for counterexamples) is Azencott [A]. Generalizing results of Feller and Hille in the case $M \subset R^{1}$, he gives conditions based on integrals involving coefficients $A, B, C$ in the expression

$$
L u(\rho)=A u^{\prime \prime}(\rho)+B u^{\prime}(\rho)+c u(\rho) .
$$

where $\rho \rightarrow \infty$ as $x \rightarrow \infty$. The conditions seem closely related to ours, yet not directly comparable.

More comparable are the results of Yau [Y]. He assumes $c=0$ in (1) and assumes a function $\rho$ such that $\rho(x) \rightarrow \infty$ as $x \rightarrow \infty$, while $L \rho \leqslant k,|d \rho|=o(\rho)$, and then constructs a kernel $p$ with $\int p=1$. (According to Theorem 1, this is the same as the minimal solution if $\rho$ is $C^{2}$.) He shows further that if $V=0$, then the solution preserves $C_{0}$ (continuous functions vanishing at $\infty$ ). His conditions on the coefficients of $L$ are slight, and $\rho$ may be just Hölder continuous, with $L \rho \leqslant k$ valid in the sense of distributions. He notes that the result applies when $M$ is complete and Ric (the Ricci curvature) is bounded below.

Dod riuk [D] gives very similar results by more elementary methods. Karp and Li $[\mathbf{K L}]$ get nearly optimal results of this type for the case $L=\Delta$. If $r$ denotes distance from a fixed point, they show that

$$
\text { Ric } \geqslant-k\left(r^{2}+1\right) \quad \Rightarrow \quad \operatorname{vol}\{r \leqslant R\} \leqslant e^{c \cdot R^{2}} \Rightarrow \text { uniqueness }
$$

and

$$
\text { Ric } \geqslant-k\left(r^{2}+1\right) \quad \Rightarrow \quad C_{0} \text { is preserved. }
$$


Our conditions are of the same type as Yau's. For uniqueness of solutions of (2), we require a positive $C^{2}$ function $\rho$ such that $\rho(x) \rightarrow \infty$ as $x \rightarrow \infty$, with $L \rho \leqslant k \rho$ (Theorem 1). If $\rho$ is the distance $r_{p}$ from a fixed point $P$ and $M$ is complete, then the inequality need hold only within the cut locus of $p$. As Li mentions in a letter, this condition follows from Ric $\geqslant-C\left(1+r_{p}^{2}\right)$; see the Appendix.

For preservation of $C_{0}$ we assume $\Delta \rho-V \rho \geqslant-k \rho$ and $|d \rho| \leqslant k \rho$ (which implies $M$ is complete). This allows us to recover Karp's and Li's result involving the Ricci curvature, but only on a manifold $M$ with a "pole", a point $p$ where the exponential map is a diffeomorphism: $M_{p} \rightarrow M$.

We are indebted to Peter $\mathrm{Li}$ and Robert Strichartz for some very helpful correspondence; to C. L. Terng for explaining the relation between Ric and bounds on $\Delta r$; to Walter Rosenkrantz for several discussions, and for bringing up the Ornstein-Uhlenbeck example (the original motivation of the paper); and to Freda Bennett for sharing her proof of a uniqueness theorem, which suggested a considerable simplification of Theorem 1 .

1. Uniqueness. First we consider whether (3) is the only solution of (2).

THEOREM 1. Let $L u=\Delta u-V u-c u$ (where $c$ has any sign) and suppose $M$ carries $a C^{2}$ function $\rho>0$ such that $\rho(x) \rightarrow \infty$ as $x \rightarrow \infty$ (in the one-point compactification) and $L \rho \leqslant k \rho$ for some constant $k$. Then

$$
u_{t}=L u,\left.\quad u\right|_{t=0}=0, \quad|u| \leqslant e^{a t} \quad \text { for some } a
$$

implies that $u \equiv 0$.

Proof. Let $w=e^{-K t} u \rho^{-1}$ with $K>\max (a, k)$. Then $w \rightarrow 0$ uniformly as $(x, t)$ $\rightarrow \infty$ and

$$
w_{t}=\Delta w-V w+2 \frac{d w \cdot d \rho}{\rho}-\left(K-\frac{L \rho}{\rho}\right) w .
$$

Since $K-(L \rho) \rho^{-1}>0$, the maximum principle shows $w=0$. In fact, if $w \neq 0$ then there is a positive maximum or a negative minimum. Suppose, say, $w\left(x_{0}, t_{0}\right)$ is a positive maximum. Then at $\left(x_{0}, t_{0}\right), 0=w_{t}=d w=V w$, while $\Delta w \leqslant 0$ and $-(K-L \rho / \rho) w<0$, contradicting $(1.1)$.

REMARKs. In case $c \geqslant 0$, the existence of a $\rho$ as in Theorem 1 guarantees that the minimal solution (3) is the only solution of (2).

In case $c=0$, then $f \equiv 1$ admits the solution $u \equiv 1$. When Theorem 1 applies, this is the only bounded solution; hence

$$
u(x, t)=\int p(t, x, y) d v_{y}=1 \text { for } t>0 .
$$

This implies that the adjoint problem is conservative, i.e. solutions of $L^{*} u=u_{t}$, have $\int_{M} u$ independent of $t$. (For the adjoint problem, $u$ is heat per unit volume.)

The condition $L \rho \leqslant k \rho$ cannot be replaced by $L \rho \leqslant k \rho^{1+\varepsilon}$ for any $\varepsilon>0$. For example, with $M=(-1,1), u=u^{\prime \prime}$, and $\rho(x)=\left(1-x^{2}\right)^{-2 / \varepsilon}$, we have $L \rho \leqslant k \rho^{1+\varepsilon}$. But in this case there are two-well known distinct solutions of (2), one with $u( \pm 1, t)=0$, and another with $u_{x}( \pm 1, t)=0$. 
Thinking in terms of heat, the condition $\Delta \rho-V \rho-c \rho \leqslant k \rho$ means that what happens at $\infty$ (the boundary of $M$ ) cannot diffuse to the finite part of $M$; note that the term $-V \rho$ gives the rate of drift in from $\infty$.

Theorem 1 applies to the generalized Ornstein-Uhlenbeck process in $R^{n}$, where

$$
L=\Delta-l(x) \cdot \nabla
$$

with $l(x)$ linear in $x$. Here $V=l(x)$ could represent a rotation, expansion, dilation, shear, etc. Taking $\rho(x)=1+|x|^{2}$, Theorem 1 guarantees uniqueness of solutions, and conservation.

In geometric applications the function $\rho$ in Theorem 1 is generally taken to be (essentially) the distance $r_{p}$ from a fixed point $p$ in $M$. If $p$ has a "cut locus" there are difficulties which can be avoided by a device due to Calabi [C]; see also [D].

THEOREM 1a. Suppose $M$ is complete and, for some point $p, L r_{p} \leqslant k r_{p}$ inside the cut locus of $p$ and for $r_{p} \geqslant \delta$. Then the conclusion of Theorem 1 holds.

PROOF. Let $\rho=r_{p}$ for $r_{p} \geqslant \delta$, with $\rho>0$ and smooth except on the cut locus of $p$. Then $L \rho \leqslant k \rho$, perhaps with a new $k$. Let $w=e^{-K t} u \rho^{-1}$ as before, with $K>k$. If $u \neq 0$ then $w$ has, say, a positive maximum $w\left(x_{0}, t_{0}\right)$. If $x_{0}$ is not on the cut locus then $r_{p}$ is smooth at $x_{0}$, and (1.1) gives a contradiction as before. If the maximum is achieved only with $x_{0}$ on the cut locus, let $\gamma$ be a minimal geodesic from $p$ to $x_{0}$. Let $q$ be on $\gamma$ at a small distance $\varepsilon>0$ from $p$. On the segment of $\gamma$ between $q$ and $x_{0}$, $r_{p}=r_{q}+\varepsilon$; and everywhere else $r_{p} \leqslant r_{q}+\varepsilon$ by the triangle inequality. So the function

$$
w_{q}=e^{-K t} u\left(r_{q}+\varepsilon\right)^{-1}
$$

has a maximum at $\left(x_{0}, t_{0}\right)$. At this point $r_{q}$ is smooth, and when $\varepsilon$ is small then

$$
L\left(r_{q}+\varepsilon\right) /\left(r_{q}+\varepsilon\right)<K
$$

in a neighborhood of $x_{0}$. So again (1.1) gives a contradiction, with $\rho=r_{q}+\varepsilon$.

\section{Vanishing at $\infty$.}

THEOREM 2. Let $L=\Delta-V-c$ with $c \geqslant 0$. Then $L$ is the generator of a contraction semigroup on $C_{0}$ if there is a Hölder continuous function $\rho$ such that as $x \rightarrow \infty$ then $\rho(x) \rightarrow \infty$ and

$$
\begin{gathered}
|d \rho| \leqslant k \rho \quad(k \text { constant }), \\
V \rho \leqslant \Delta \rho+k \rho \quad \text { in the sense of distributions. }
\end{gathered}
$$

REMARKS. (2.1) implies $M$ is complete, and (2.2) says the drift to $\infty$ is not too rapid. A simple application of Theorem 2 is the Ornstein-Uhlenbeck process (1.2).

The proof uses a version of the Lumer-Phillips Theorem [Yo]: A closed operator $L$ on a Banach space $B$ is the generator of a contraction semigroup $e^{L t}$ if and only if $L$ is dissipative and $L^{*}$ has no positive eigenvalue. Dissipative means that for every $u$ in the domain of $B$ there is a $\tilde{u}$ in $B^{*}$ with

$$
\|\tilde{u}\|=1, \quad\langle u, \tilde{u}\rangle=\|u\|, \quad \text { and }\langle L u, \tilde{u}\rangle \leqslant 0 .
$$


This implies that the range of $\lambda I-L$ is closed for $\lambda>0$, and the lack of positive eigenvalues for $L^{*}$ then implies $\lambda I-L$ is surjective.

Lemma 2.1. $L$ is dissipative on the domain $\left\{u\right.$ in $C_{0}: L u$ in $\left.C_{0}\right\}$ iff $c \geqslant 0$.

This is more or less well known, but for completeness we prove the "if" part.

On $C_{0}$ the vector $\tilde{u}$ is a unit measure concentrated at a maximum point $x_{0}$ for $u$, or minus a unit measure concentrated at a minimum point. Suppose the first case. Since $L u \in C_{0}$, the elliptic regularity theorem shows that $u$ is $C^{2-\varepsilon}$ for every $\varepsilon>0$; hence $V u$ and, with it, $\Delta u=L u+V u+c u$ are continuous. Since $x_{0}$ is a maximum point, $d u\left(x_{0}\right)=0$ and

$$
\langle L u, \tilde{u}\rangle=(L u)\left(x_{0}\right)=\Delta u\left(x_{0}\right)-c\left(x_{0}\right) u\left(x_{0}\right) \leqslant \Delta u\left(x_{0}\right),
$$

since we assume $c \geqslant 0$, and $u\left(x_{0}\right) \geqslant 0$ at the maximum of any function in $C_{0}$. By an argument as in Courant and Hilbert [CH, leading up to p. 286], any continuous function $u$ such that $\Delta u$ is also continuous satisfies

$$
\Delta u\left(x_{0}\right)=\lim _{R \rightarrow 0} \frac{2 n}{\left|S^{n-1}\right|} R^{-1-n} \int_{d\left(x, x_{0}\right)=R}\left[u(x)-u\left(x_{0}\right)\right] d \sigma,
$$

where $\left|S^{n-1}\right|$ is the area of the $n-1$ sphere, $d\left(x, x_{0}\right)$ is geodesic distance, and $d \sigma$ is the induced measure on the sphere $\left\{d\left(x, x_{0}\right)=R\right\}$. Since $u\left(x_{0}\right)$ is a maximum, it follows that $\Delta u\left(x_{0}\right) \leqslant 0$, and $L=\Delta-V-c$ is dissipative, proving Lemma 2.1.

It remains to prove that $L^{*}$ has no positive eigenvalue. Suppose $\mu$ is a finite measure such that $L^{*} \mu=\lambda \mu, \lambda>0$. By regularity, $d \mu=u d v$, where $d v$ is the Riemann volume element, and $u$ is a $C^{2}$ function, in $L^{1}(d v)$ since $\mu$ is a finite measure, satisfying $L^{*} u=\lambda u$. Let $\psi$ be a $C^{2}$ function on the line, $\psi \geqslant 0, \psi(\rho)=1$ for $0 \leqslant \rho \leqslant 1, \psi(\rho)=0$ for $\rho \geqslant 2, \psi^{\prime} \leqslant 0$. Set

$$
\phi_{m}(x)=\psi(\rho / m) \text {. }
$$

Since $L^{*} u=\lambda u$ with $\lambda>0$,

$$
\begin{aligned}
0 & \leqslant \lambda \int \phi_{m} u \operatorname{sgn} u=\int \phi_{m}\left(L^{*} u\right) \operatorname{sgn} u \\
& \leqslant \int \phi_{m}\left(\Delta u-V^{*} u\right) \operatorname{sgn} u \quad[\text { since } c \geqslant 0] \\
& \leqslant \int\left(\Delta \phi_{m}-V \phi_{m}\right)|u|
\end{aligned}
$$

by Kato's inequality [K]. Further,

$$
\Delta \phi_{m}-V \phi_{m}=\frac{\rho^{2}}{m^{2}} \psi^{\prime \prime} \frac{|d \rho|^{2}}{\rho^{2}}+\frac{\rho}{m} \psi^{\prime}\left(\frac{\rho}{m}\right) \frac{\Delta \rho-V \rho}{\rho} \leqslant K
$$

in view of (2.1) and (2.2), since $\psi^{\prime} \leqslant 0$. The integrand tends to 0 and is dominated by a constant times $|u|$, so $\lambda \int|u|=0$. Hence, $u=0$ and Theorem 2 is proved. 
3. The $L^{p}$ case. It is fair to ask: $L^{p}$ with respect to what measure? We allow a general $C^{1}$ measure on $M$ which, in local coordinates, is $m d x$, with $m$ in $C^{1}$. Our operator can be written

$$
\begin{aligned}
L & =\frac{1}{m} \sum \frac{\partial}{\partial x_{j}} g^{j k} m \frac{\partial}{\partial x_{k}}-\sum b_{j} \frac{\partial}{\partial x_{j}}-c \\
& =A-V_{m}-c,
\end{aligned}
$$

where $g^{j k}, b_{j}, c$, are sufficiently smooth, and the matrix $\left(g^{j k}\right)$ is positive definite. defining a metric on the cotangent bundle. Define inner products

$$
\langle u, v\rangle=\int u v m d x
$$

and, for 1-forms,

$$
\langle u, v\rangle=\int(u, v) m d x,
$$

where $(u, v)$ is the inner product in the cotangent bundle. Define the " $m$-divergence" of the vector field

$$
V=\sum b_{i} \frac{\partial}{\partial x_{j}}
$$

as

$$
\nabla_{m} V=\frac{1}{m} \sum \frac{\partial b_{j} m}{\partial x_{j}}
$$

Then for $u, w$ in $C_{c}^{2}$,

$$
\begin{aligned}
& \langle A u, w\rangle=-\langle d u, d w\rangle=\langle u, A w\rangle, \\
& \langle V u, w\rangle=-\langle u, V w\rangle-\left\langle u,\left(\nabla_{m} V\right) w\right\rangle=\left\langle u, V^{*} w\right\rangle .
\end{aligned}
$$

These formulas show that the representation of $A$, and the " $m$-divergence", are coordinate invariant.

THEOREM 3. For $1 \leqslant p \leqslant \infty$, $L$ is dissipative on the domain $C_{c}^{2} \subset L^{p}$ if

$$
(1 / p) \nabla_{m} V_{m} \leqslant c .
$$

Hence, $L$ is also dissipative on the closure of this domain in graph norm.

Proof. Let $u \in C_{c}^{2}, p>1$. Then $u d|u|=|u| d u$ so

$$
d\left(|u|^{p-2} u\right)=(p-1)|u|^{p-2} d u .
$$

Take $\tilde{u}=|u|^{p-2} u$, and compute

$$
\begin{aligned}
\left\langle A u-\frac{1}{p} V_{m} u-c u,|u|^{p-2} u\right\rangle= & -(p-1)\left\langle d u,|u|^{p 2} d u\right\rangle+\frac{1}{p}\left\langle u, V_{m}\left(u|u|^{p 2}\right)\right\rangle \\
& +\frac{1}{p}\left\langle u,\left(\nabla_{m} V_{m}\right) u|u|^{p 2}{ }^{2}\right\rangle-\left\langle c u, u|u|^{p-2}\right\rangle .
\end{aligned}
$$


But

$$
\frac{1}{p}\left\langle u, V_{m}\left(u|u|^{p-2}\right)\right\rangle=\frac{p-1}{p}\left\langle u,(V u)|u|^{p-2}\right\rangle,
$$

so canceling and transposing gives

$$
\begin{aligned}
\left\langle A u-V_{m} u\right. & \left.-c u,|u|^{p-2} u\right\rangle \\
& =-(p-1)\left\langle d u,|u|^{p-2} d u\right\rangle+\left\langle\left(\frac{1}{p} \nabla_{m} V_{m}-c\right) u, u|u|^{p-2}\right\rangle \\
& \leqslant 0 \quad \text { if }\left(\mathrm{D}_{p}\right) \text { holds. }
\end{aligned}
$$

For $p=1$, take the limit in the last equality as $p \rightarrow 1$. The first term remains $\leqslant 0$, and the limit of the second term is $\leqslant 0$ by $\left(D_{1}\right)$.

REMARK. The vector field $V_{m}$ represents drift. The condition $\left(\mathrm{D}_{p}\right)$ says that the divergence of the drift must be compensated by the dissipation $c$. Examples below show that this condition is sharp in simple cases where $\nabla_{m} \cdot V_{m}$ and $c$ are constant. When $p=1$, condition $\left(\mathrm{D}_{1}\right)$ is also necessary, as the last equality in the proof shows.

Finally, we give conditions eliminating positive eigenvalues for $L^{*}$, thus guaranteeing a contraction semigroup on $L^{p}$.

THEOREM 4. There are no nonzero solutions of $L^{*} u=\lambda u$ in $L^{q}(1 / p+1 / q=1)$ if

$$
\lambda \geqslant(1 / p) \nabla_{m} V_{m}-c
$$

and there is a function $\rho$ on $M$ such that

$$
\rho(\infty)=\infty, \quad|d \rho|=o(\rho), \quad\left|V_{m} \rho\right| \leqslant k \rho .
$$

Note. If $V_{m}=0$ this is in Strichartz [S], who refers also to Yau.

Proof. It suffices to take $\lambda=0$ (replace $c$ by $c-\lambda$ ), so the hypothesis is

$$
(1 / p) \nabla_{m} V_{m}-c \leqslant 0 .
$$

Let $H(u)=u|u|^{q-2}$. For $\phi$ in $C_{c}^{2}$ consider

$$
\begin{aligned}
I= & \left\langle\phi^{2} H(u), A u-\frac{1}{q} V_{m}^{*} u-c u\right\rangle \\
= & -\left\langle d\left(\phi^{2} H\right), d u\right\rangle-\frac{1}{q}\left\langle V_{m}\left(\phi^{2} H\right), u\right\rangle-\left\langle c \phi^{2} H, u\right\rangle \\
= & -2\langle\phi H d \phi, d u\rangle-\left\langle\phi^{2} H^{\prime} d u, d u\right\rangle-\frac{1}{q}\left\langle\phi^{2}\left(V_{m} u\right) H^{\prime}, u\right\rangle-\frac{2}{q}\left\langle\phi H V_{m} \phi, u\right\rangle \\
& -\frac{1}{q}\left\langle\phi^{2}\left(\nabla_{m} V_{m}\right) u H^{\prime}, u\right\rangle+\frac{1}{q}\left\langle\phi^{2}\left(\nabla_{m} V_{m}\right) u H^{\prime}, u\right\rangle-\left\langle\phi^{2} c H, u\right\rangle .
\end{aligned}
$$

But $u H^{\prime}=(q-1) H$ and $V_{m}^{*} u=-V_{m} u-\nabla_{m} V_{m} u$, so

$$
\begin{aligned}
I= & -2\langle\phi H d \phi, d u\rangle-\left\langle\phi^{2} H^{\prime} d u, d u\right\rangle+\frac{1}{p}\left\langle\phi^{2} H, V_{m}^{*} u\right\rangle \\
& -\frac{2}{q}\left\langle\phi H V_{m} \phi, u\right\rangle+\frac{1}{p}\left\langle\phi^{2}\left(\nabla_{m} V_{m}\right) H, u\right\rangle-\left\langle\phi^{2} c H, u\right\rangle .
\end{aligned}
$$


Transpose $(1 / p)\left\langle\phi^{2} H, V_{m}^{*} u\right\rangle$, use $L^{*} u=0$ and (3.2) to get

$$
0=\left\langle\phi^{2} H, L^{*} u\right\rangle \leqslant-2\langle\phi H d \phi, d u\rangle-\left\langle\phi^{2} H^{\prime} d u, d u\right\rangle-\frac{2}{q}\left\langle\phi H V_{m} \phi, u\right\rangle .
$$

But $H^{\prime}=(q-1)|u|^{q-2}$, so

$$
\begin{aligned}
(q-1)\left\|\phi|u|^{q / 2-1} d u\right\|^{2} \leqslant & 2 \sup |d \phi| \cdot\left\|\phi|u|^{q / 2-1} d u\right\| \cdot\left\|u^{q / 2}\right\| \\
& -\frac{2}{q}\left\langle\phi H V_{m} \phi, u\right\rangle,
\end{aligned}
$$

where all norms are in $L^{2}$. Now let $\phi=\psi(\rho / j)$, with $\psi$ as in the proof of Theorem 2 . Then

$$
u \phi H V_{m} \phi=|u|^{q} \psi\left(\frac{\rho}{j}\right) \frac{\rho}{j} \psi^{\prime}\left(\frac{\rho}{j}\right)\left(\frac{V_{m} \rho}{\rho}\right) .
$$

By hypothesis, $\left|V_{m} \rho\right| \leqslant k \rho$ and $u \in L^{q}$, so by dominated convergence,

$$
\left\langle\phi H V_{m} \phi, u\right\rangle \rightarrow 0 \quad \text { as } j \rightarrow \infty .
$$

Also, as $j \rightarrow \infty$,

$$
|d \phi|=\frac{\rho}{j} \psi^{\prime}\left(\frac{\rho}{j}\right)\left(\frac{|d \rho|}{\rho}\right) \rightarrow 0
$$

uniformly, since $|d \rho| / \rho \rightarrow 0$ as $\rho \rightarrow \infty$. Hence, from (3.3),

$$
\left\|\phi|u|^{q / 2-1} d u\right\| \rightarrow 0 .
$$

It follows that $u$ is constant on any open set where $u \neq 0$. Since $u$ is continuous and in $L^{q}, u \equiv 0$. Q.E.D.

When the conditions in Theorems 3 and 4 are met for two values $p_{1}<p_{2}$, the resulting semigroups agree on $L^{p_{1}} \cap L^{p_{2}}$. The proof follows Strichartz [S], showing that the resolvent $(L-\lambda)^{-1}$ is the same in $L^{p_{1}}$ as in $L^{p_{2}}$ for large $\lambda$. If the resolvents are different, there is a $u_{1}$ in $L^{p_{1}}$ and a $u_{2}$ in $L^{p_{2}}$ with $u_{1} \neq u_{2}$ and

$$
(L-\lambda) u_{1}=(L-\lambda) u_{2}
$$

so $u_{1}-u_{2}$ is an eigenvalue of $L$ in $L^{p_{1}}+L^{p_{2}}$. We prove that the difference $u=u_{1}-u_{2}=0$ if

$$
\frac{1}{p} \nabla_{m} V_{m}-c \leqslant \lambda, \quad \text { for } p=p_{1}>1 \text {, and } p=p_{2}>p_{1} \text {. }
$$

The proof imitates Theorem 4 with an appropriate choice of $H$. Choose an increasing smooth function $\theta$ with

$$
\theta(u)= \begin{cases}1 / p_{2}, & 0 \leqslant u \leqslant 1, \\ 1 / p_{1}, & 2 \leqslant u\end{cases}
$$

Define an even function $G$ with

$$
\begin{aligned}
G(u) & =\frac{1}{u} \int_{0}^{u} \theta=\int_{0}^{1} \theta(t u) d t, \quad u>0, \\
& =\left\{\begin{array}{l}
1 / p_{2}, \quad 0 \leqslant u \leqslant 1, \\
1 / p_{1}-c_{1} / u, \quad 2 \leqslant u,
\end{array}\right.
\end{aligned}
$$


$G^{\prime}(u)=\int_{0}^{1} t \theta^{\prime}(t u) d t \geqslant 0$, so $1 / p_{2} \leqslant G \leqslant 1 / p_{1}$. Hence (3.4) gives

$$
G(u) \nabla_{m} V_{m}-c \leqslant \lambda \text {. }
$$

Next define $H$ odd with

$$
\begin{aligned}
H(u) & =\left(p_{2} G u\right)^{-1} \exp \left(\int_{1}^{u}(G s)^{-1} d s\right), \quad u>0, \\
& =\left\{\begin{array}{l}
u^{p_{2}-1}, \quad 0 \leqslant u \leqslant 1, \\
c_{2}\left(u-p_{1} c_{1}\right)^{p_{1}-1}, \quad 2 \leqslant u .
\end{array}\right.
\end{aligned}
$$

Then

$$
G H+(G H)^{\prime} u=H
$$

so $H^{\prime}=\left[1-(G u)^{\prime}\right] H / G u=[1-\theta] H / G u>0$. Hence further

$$
\sqrt{H / u} \leqslant \text { const } \sqrt{H^{\prime}} \text {. }
$$

Now suppose that $L u=\lambda u$ with $u \in L^{p_{1}}+L^{p_{2}}$. Then $u H(u)$ is in $L^{1}$. Consider

$$
I=\left\langle\phi^{2} H(u), A u-G(u) V_{m} u-(c+\lambda) u\right\rangle
$$

and calculate as before, using $(3.4 \mathrm{a}, \mathrm{b})$, to get

$$
\left\langle\phi^{2} H^{\prime} d u, d u\right\rangle \leqslant-2\langle\phi H d \phi, d u\rangle+2\left\langle G H \phi V_{m} \phi, u\right\rangle .
$$

Thus

$$
\left\|\phi \sqrt{H^{\prime}} d u\right\|^{2} \leqslant 2 \sup |d \phi| \cdot\|\phi \sqrt{H / u}\| \cdot\|\sqrt{H u}\|+2\left\langle G H \phi V_{m} \phi, u\right\rangle .
$$

Applying (3.4c) and assuming a $\rho$ as in Theorem 4, we find as before that $u \equiv 0$. This proves that the semigroups in $L^{p_{1}}$ and in $L^{p_{2}}$ agree on $L^{p_{1}} \cap L^{p_{2}}$. [Note: The above result is a revision in the galley proofs of a weaker result in the original article.]

We conclude with two examples. The first shows that the condition $(1 / p) \nabla_{m} V_{m}-$ $c \leqslant \lambda$ in Theorem 4 is essential, as is $c-\nabla_{m} V_{m} \geqslant 0$ in Theorem 3.

EXAMPLE 1. $L u=u^{\prime \prime}-b x u^{\prime}$, where $b$ is constant; take $m d x=d x$. (When $b<0$, this is the Uhlenbeck process.)

The Fourier transform with respect to $x$ converts $L u=u_{t}$ into the first order equation

$$
-\xi^{2} \hat{u}+b \frac{\partial}{\partial \xi}(\xi \hat{u})=\frac{\partial \hat{u}}{\partial t} .
$$

The solution with $\hat{u}(0, \xi)=\hat{f}(\xi)$ is

$$
\hat{u}(t, \xi)=\hat{f}\left(\xi e^{b t}\right) \exp \left(b t-\left(\xi^{2} / 2 b\right)\left(e^{2 h t}-1\right)\right) .
$$

Taking a limit as $b \rightarrow 0$ gives the usual solution of $u^{\prime \prime}=u_{t}$.

If this is a contraction on $L^{p}$, then the adjoint $L^{*}$ has no positive eigenvalue in $L^{q}$. The eigenvalue equation $L^{*} u=\lambda u$ is solved by taking the Fourier transform:

$$
\hat{u}(\xi)=|\xi|^{-\lambda / h} e^{-\xi^{2} / 2 b}
$$

If $b<0$ there is no solution in $L^{q}$. If $b>0$ we have

$$
u(x)=c|x|^{-1+\lambda / b} * e^{-b x^{2} / 2}
$$


which is in $L^{q}$ iff $q(-1+\lambda / b)<-1$, that is iff $\lambda<b / p$, where $1 / p+1 / q=1$.

Combined with Theorems 3 and 4 , this shows that $L-c$ (where $c$ is constant) generates a contraction semigroup on $L$ iff $c-b / p \geqslant 0$. Note that in this case $b=\nabla_{m} V_{m}$, so Theorems 3 and 4 are sharp.

EXAmple 2. $L u=\Delta u-b\left(x u_{y}-y u_{x}\right) ; m$ is Lebesgue measure on $R^{2}$. Here $\nabla_{m} V_{m}=0$, so there is a contraction semigroup on $L^{p}$ for $1<p<\infty$, and on $C_{0}$. We analyze the spectrum of $L$ on the space $L^{2}$ by taking the Fourier transform:

$$
\hat{L} v=-\left(\xi^{2}+\eta^{2}\right) v+b \eta \frac{\partial v}{\partial \xi}-b \xi \frac{\partial v}{\partial \eta} .
$$

The first order equation

$$
\hat{L} \phi=\lambda \phi
$$

gives the rotation vector field

$$
\dot{\xi}=b \eta, \quad \dot{\eta}=-b \xi,
$$

where $\dot{\xi}$ is the derivative of $\xi$ with respect to a parameter $\tau$. The flow is

$$
\xi=r \cos b \tau, \quad \eta=-r \sin b \tau, \quad r \text { constant. }
$$

The solution of (3.5) is, with $b \tau=\theta$,

$$
\phi(r \cos \theta, r \sin \theta)=e^{-\left(\lambda+r^{2}\right) \theta / h} \phi(r, 0),
$$

where we obviously need $e^{-\left(\lambda+r^{2}\right) 2 \pi / h}=1$, or

$$
\lambda+r^{2}=i b k, \quad k=0, \pm 1, \pm 2, \ldots
$$

The spectrum of $L$ consists of the union of half-lines $\left\{\lambda: \lambda=i b k-r^{2}, k=\right.$ $0, \pm 1, \ldots, r \geqslant 0\}$. This comes right down to $\operatorname{Im} \lambda=0$, so $\left\|e^{t l}\right\|=1$.

Appendix. We sketch a proof of some relations between Ricci curvature and $\Delta r$. The proof of Lemma 1 was given by C. L. Terng.

Lemma 1. Let $r$ denote distance from a fixed point $p$. If Ric $\geqslant-C\left(1+r^{2}\right)$, then $(\Delta r)_{r} \leqslant-(\Delta r)^{2} / n+C\left(1+r^{2}\right)$ inside the cut locus of $p$.

Proof. Choose an orthonormal local frame $e_{1}, \ldots, e_{n}$ with $e_{1}=\partial / \partial r$. Set $r_{i}=\nabla_{e_{1}} r$. Since $|\nabla r|^{2}=1$ and $r_{i j}=r_{j i}$,

$$
0=\Delta\left(\frac{1}{2}|\nabla r|^{2}\right)=\sum_{i}\left(\sum_{j} r_{j} r_{j i}\right)_{i}=\sum\left(r_{j i}^{2}+r_{j} r_{j i i}\right)=\sum\left(r_{i j}^{2}+r_{j} r_{i j i}\right) .
$$

The Ricci formula gives

$$
r_{i j k}=r_{i k j}+\sum_{l} r_{l} R_{l i j k},
$$

so (now summing repeated indices)

$$
r_{i j i}=r_{i i j}+r_{l} R_{l i j i}=r_{i i j}+r_{l} R_{l j},
$$

with $R_{l j}$ the Ricci tensor. So (*) gives

$$
0=\sum r_{i j}^{2}+r_{j}\left(r_{i i j}+r_{l} R_{l j}\right) .
$$


Set $f=\Delta r=r_{i i}$, and get

$$
r_{j} f_{j}=r_{j} r_{i i j}=-\sum r_{i j}^{2}-r_{j} r_{l} R_{l j} \leqslant-\sum r_{i j}^{2}+C\left(1+r^{2}\right),
$$

Since $r_{1}=1, r_{2}=\cdots=r_{n}=0, f_{1}=\partial f / \partial r$, and

$$
\sum r_{i j}^{2} \geqslant \sum r_{i i}^{2} \geqslant \frac{1}{n}\left(\sum r_{i i}\right)^{2}=\frac{1}{n}(\Delta r)^{2},
$$

the lemma follows.

LEMMA 2. If

$$
f^{\prime}(r)<-a^{2} f^{2}+b^{2}+c^{2} r^{2}, \quad r_{0}>r>0,
$$

then

$$
f \leqslant(2 / a)(1 / a r+b+c r), \quad r_{0}>r>0 .
$$

If $r_{0}=\infty$ then

$$
f \geqslant-(\rho / a) \sqrt{b^{2}+c^{2} r^{2}}
$$

as well, where $\rho$ is a constant $>1$ satisfying

$$
c \rho \leqslant a b^{2}\left(\rho^{2}-1\right) .
$$

Proof OF (2).

Case 1. Suppose that for small $r$,

$$
\sqrt{b^{2}+c^{2} r^{2}}<a f / \sqrt{2} .
$$

Then $f^{\prime}(r) \leqslant-a^{2} f^{2} / 2$, so $(1 / f)^{\prime} \geqslant a^{2} / 2$, hence, by (5), $1 / f \geqslant a^{2} r / 2$, or $f \leqslant 2 / a^{2} r$. Further, $f^{\prime}(r) \leqslant 0$ as long as $f^{2} \geqslant\left(b^{2}+c^{2} r^{2}\right) / a^{2}$. So $f$ must lie below the dashed line in Figure 1; it cannot cross the graph of $(1 / a)\left(b^{2}+c^{2} r^{2}\right)^{1 / 2}$ from below, since (1) implies $f^{\prime} \leqslant 0$ at such a crossing. So, being generous,

$$
f \leqslant \frac{2}{a^{2} r}+\frac{\sqrt{2}}{a}\left(b^{2}+c^{2} r^{2}\right)^{1 / 2} \leqslant \frac{2}{a}\left(\frac{1}{a r}+b+c r\right) .
$$

Case 2. This is even easier; $f$ starts out below the graph of $(\sqrt{2} / a)\left(b^{2}+c^{2} r^{2}\right)^{1 / 2}$.

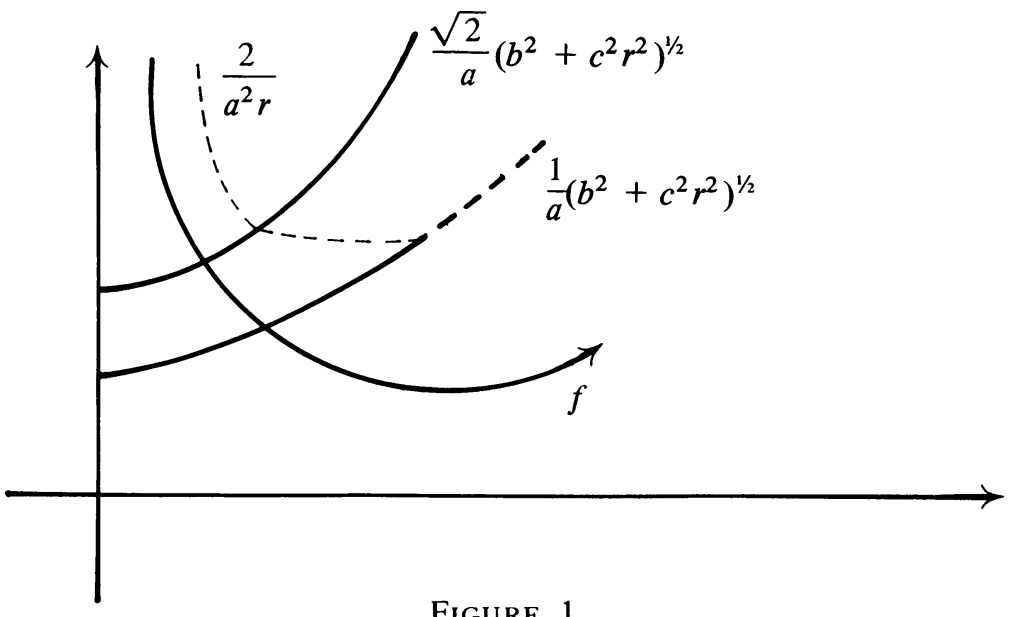

FigURE 1 
Proof of (3). Compare $f$ to the function $g(r)=-(\rho / a)\left(b^{2}+c^{2} r^{2}\right)^{1 / 2}$, with $\rho>1$ satisfying (4). From (1), $f \leqslant g \Rightarrow f^{\prime}<g^{\prime}$, so $f$ cannot cross the graph of $g$ from below; if $f\left(r_{1}\right) \leqslant g\left(r_{1}\right)$, then $f(r)<g(r)$ for all $r>r_{1}$. But $f \leqslant g$ implies

$$
f^{\prime} \leqslant-a^{2} f^{2}+b^{2}+c^{2} r^{2} \leqslant-a^{2} f^{2}+a^{2} f^{2} / \rho^{2}=-\alpha f^{2}, \quad \alpha>0 .
$$

So $(1 / f)^{\prime} \geqslant \alpha>0$, and this implies that $1 / f>0$ eventually, contradicting $f \leqslant g<0$.

Combining the lemmas with $f=\Delta r$, we find from (2) that

$$
\text { Ric } \geqslant-C\left(1+r_{p}^{2}\right) \Rightarrow \Delta r_{p} \leqslant k\left(1 / r_{p}+r_{p}\right)
$$

inside the cut locus of $p$. (This also follows from the Laplacian Comparison Theorem of Greene and Wu (Lecture Notes in Math., vol. 699, Springer-Verlag, Berlin and New York) taking as model the space $R^{n}$ with metric $d r^{2}+\exp \left(r^{2}\right) d \theta^{2}$ in polar coordinates.)

If exp: $M_{p} \rightarrow M$ is a diffeomorphism, then (3) gives

$$
\text { Ric } \geqslant-C\left(1+r_{p}^{2}\right) \Rightarrow \Delta r_{p} \geqslant-k r_{p}
$$

since $\Delta r \rightarrow+\infty$ as $r \rightarrow 0$. (This inequality does not seem to follow from the theorem of Greene and Wu.) Hence Theorem 2 applies: $C_{0}$ is preserved.

\section{REFERENCES}

[A] R. Azencott, Behaivior of diffusion semigroups at infinity, Bull. Soc. Math. France 102 (1974), $193-240$.

[C] E. Calabi, An extension of E. Hopf's maximum principle with an application to geometry, Duke Math. J. 25 (1958), 45-56.

[CH] R. Courant and D. Hilbert, Methods of mathematical physics, Vol. 2, Interscience, New York, 1962.

[D] J. Dodziuk, Maximum principle for parabolic inequalities and the heat flow on open manifolds, Indiana Univ. Math. J. 32 (1983), 703-716.

[K] T. Kato, Schrödinger operators with singular potentials, Israel J. Math. 13 (1972), 135-148.

[KL] Leon Karp and Peter Li, The heat equation on complete Riemannian manifolds (preprint).

[S] R. Strichartz, Analysis of the Laplacian on a complete Riemannian manifold, Cornell Univ. (preprint).

[Y] S. T. Yau, On the heat kernel of a complete Riemannian manifold, J. Math. Pures Appl. (9) 57 (1978), $191-201$.

[Yo] K. Yosida, Functional analysis, Springer, New York, 1974.

Department of Mathematics, University of Massachusetts, Boston, Massachusetts 02125 